\title{
De madres a hijas, de hijas a madres: El cambio en la transmisión intergeneracional de lenguas
}

\author{
Julia Llompart Esbert, Universitat Autònoma de Barcelona \\ Barcelona, España
}

Artículo recibido el 3 de diciembre de 2013, aceptado el 19 de febrero de 2013, versión final recibida el 22 de julio 2013

\begin{abstract}
Este trabajo pretende investigar los cambios en la dinámica de la transmisión de lenguas que se produce en familias procedentes de Pakistán y Marruecos y, en concreto, entre madres e hijas, éstas últimas en edades escolares y escolarizadas, total o parcialmente, en Cataluña. Nos interesa especialmente analizar las concepciones familiares y lingüísticas que tienen estas niñas sobre sus madres y, por lo tanto, las categorizaciones que hacen de sus progenitoras y, también, las auto-categorizaciones de sus madres. Para ello, se analizan los contenidos de conversaciones, en el ámbito escolar y fuera de la escuela entre las jóvenes y entre las jóvenes y sus madres, y se relacionan con los estudios sobre la socialización lingüística, las categorizaciones y la construcción de la identidad. Esta investigación se centra en estas niñas y jóvenes que "dicen hacer de maestras de sus progenitores o hermanos" (Unamuno y Nussbaum, 2006), con el objetivo de descubrir cómo se produce esta transmisión de la lengua y cómo es su competencia plurilingüe.
\end{abstract}

\section{Introducción}

La realidad escolar actual en Cataluña se ve influenciada por el fenómeno social migratorio que se inició durante los años noventa del siglo pasado y que ha provocado que, en algunos centros escolares, el porcentaje de alumnado inmigrante sea del $90 \%$. Los inmigrantes del siglo XXI, a diferencia de los de la ola migratoria del sur de España de los años cincuenta y sesenta, son marroquíes, ecuatorianos, peruanos, colombianos, chinos y pakistaníes, personas que llegan de las zonas más empobrecidas del mundo (Unamuno y Codó, 2007). La imagen de la escuela catalana actual es multicultural y multilingüe, cosa que plantea nuevos interrogantes para dilucidar a través de la observación de los recientes procesos sociolingüísticos y socioculturales.

Para poder llevar a cabo una educación de calidad frente a la nueva realidad, hay que analizar cómo funciona el modelo lingüístico educativo de Cataluña y cómo es la socialización lingüística de los niños y jóvenes de origen inmigrante en las lenguas de la sociedad de acogida como han hecho estudios anteriores (Nussbaum y Unamuno, 2006, Unamuno y Nussbaum, 2006, Unamuno y Codó, 2007, Cots y Nussbaum, 2008). Este estudio pretende ser una aportación a los trabajos realizados anteriormente y se centra en el análisis del proceso de (re)socialización en una nueva sociedad a través de la o las lenguas, así como también en el análisis de la transmisión de las lenguas. 
Este estudio se realizó en dos centros educativos de Barcelona, uno de educación primaria $^{1}$ (barrio de Sant Pere) y uno de educación secundaria (barrio del Raval) en los cuales hay un gran número de alumnado de origen inmigrante (de primera y de segunda generación). El trabajo toma un enfoque etnográfico para ver cómo son los procesos de construcción de la identidad, de integración social y de desarrollo de actitudes, ideologías y usos lingüísticos por parte de niñas y madres, y tratar en profundidad el tema de la socialización lingüística y la construcción interactiva de la identidad a través del análisis del discurso. Además, el trabajo se inscribe dentro del campo del biplurilingüismo y del desarrollo de la conciencia metalingüística, puesto que las niñas participantes del estudio son plurilingües $\mathrm{y}$, por lo tanto, susceptibles de desarrollar una capacidad específica de expertas y de transmisoras de lengua, capacidades que requieren una cierta competencia de reflexión metalingüística.

Para todo ello, realizaremos en primer lugar una revisión de los estudios relacionados con las diferentes áreas específicas que nos interesan (socialización lingüística, identidad, categorizaciones, conciencia metalingüística y language brokerage); posteriormente, presentaremos los datos y la información sobre las participantes en el estudio de manera sucinta, así como la metodología empleada para su análisis; finalmente, se mostrarán los resultados del análisis y se discutirán.

\section{Los factores de la nueva socialización lingüística}

Uno de los marcos centrales sobre el cual se apoya este trabajo es el de las teorías de la socialización lingüística (SL, de ahora en adelante) desarrolladas durante los años ochenta por Schieffelin y Ochs (Ochs y Schieffelin, 1982; Schieffelin y Ochs, 1986) para dar respuesta a dos vacíos: por un lado, el que dejan los estudios psicolingüísticos de la adquisición de lenguas, los cuales no incluyen el factor cultural, y, por otro lado, las carencias observadas en los estudios antropológicos de socialización de niños y niñas, que no contemplan los aspectos lingüísticos de la socialización. Según Kulick y Schieffelin (2004), el paradigma de la SL da respuesta a la falta del elemento cultural en la investigación sobre la adquisición de lenguas y, de igual manera, a la ausencia de la lengua en los estudios sobre socialización. Ambos aspectos son relevantes ya que los niños y niñas, para convertirse en miembros competentes de un determinado grupo social, se tienen que socializar a través de la lengua y para usar la lengua. La 
investigación en SL quiere mostrar que toda interacción humana es socializadora y que hay que prestar atención a la manera cómo las subjetividades se negocian, es decir, que no son dadas de forma automática (Kulick y Schieffelin, 2004) y que no son unilaterales; en una interacción madre-hijo, no sólo la madre socializa al hijo en tanto que hijo a través de sus acciones y verbalizaciones, sino que ella es también socializada como madre. Investigadoras como Schieffelin y Ochs (1986) afirman que el análisis del uso de la lengua (o las lenguas) por parte tanto de niños como de adultos para expresar relaciones (con el conjunto de la sociedad, grupos o personas concretos) permite observar cómo se construyen las subjetividades a través de la interacción social.

El presente estudio se enmarca en la realidad multicultural y multilingüe de dos barrios de Barcelona. Los datos son básicamente un conjunto de entrevistas y interacciones entre niñas, entre adolescentes y entre adolescentes y sus madres, recogidos tanto dentro del ámbito escolar como fuera. El análisis de los datos nos sirve para estudiar cómo las hijas y las madres aprenden la lengua (o las lenguas) y qué uso hacen tanto de las lenguas autóctonas como alóctonas. El análisis de la interacción social y el descubrimiento de la construcción de las identidades de las madres y las hijas nos ayuda a entender cómo es la SL de unas y de otras. Además, podremos ver si en la SL de las hijas tiene un papel importante la familia o si son las hijas quienes, por el hecho de estar escolarizadas en Cataluña y en catalán y castellano, participan activamente en la resocialización lingüística de sus madres. Según Schieffelin y Ochs (1986), 'el conocimiento cultural y las creencias se transmiten de generación en generación y en la interacción diaria', pero, los datos del presente estudio permitirán mostrar si la transmisión de lengua, que lleva implícito el conocimiento sobre su uso en una cultura concreta, sigue una única dirección (de madres a hijas) o si es bidireccional (las lenguas alóctonas son transmitidas de madres a hijas y, las lenguas autóctonas de hijas a madres). Éste es un fenómeno que ocurre en procesos de migración y que tiene unas características específicas y unas consecuencias tanto para las madres como para las hijas. Bayley y Schecter (2003), que se fijan en las nuevas realidades bilingües y multilingües en el contexto migratorio latino en los Estados Unidos, entienden la SL como un proceso que se da toda la vida y en el cual aquéllos y aquéllas que son socializados, especialmente en contextos en los cuales conviven más de una lengua, tienen, además, la oportunidad de elegir. Incorporan asimismo el discurso sobre la 
identidad como elemento que se crea en un nuevo contexto y en la SL. La teoría de la performance (Butler, 1990; Fisher, 1970; Lakoff 1975; Miller 2012) parte de la idea de que, en un contexto determinado, se crean nuevas subjetividades basadas en las interacciones, en el discurso y en la actuación de los sujetos de estudio. Según Fischer, entonces, la lengua se aprendería para 'mantener y cambiar de forma apropiada y progresiva la posición como miembros de una sociedad' (Fisher 1970, pp. 107-108) y se haría, como hemos visto, de forma interactiva, a través del uso social de las lenguas.

\section{Sujetos, identidades y categorizaciones}

En este estudio nos interesa especialmente cómo se perciben e identifican las participantes y las relaciones que se establecen entre ellas en relación a la lengua, a la sociedad en la que viven, a sus familias, etc. Ello precisa ser observado desde diferentes puntos de vista. En primer lugar, podemos definirlas como sujetos de la sociedad. Según Butler (1990), un sujeto es una persona:

sometida a un conjunto de reglas sociales, ... la ley que dirige esas reglas es, por un lado, el principio formativo del sexo, el género, los placeres y los deseos de la persona $\mathrm{y}$, por otro lado, es el principio hermenéutico de la autointerpretación. (p. 96)

En esta línea de condicionamiento y construcción social del sujeto irían las propuestas constructivistas de muchos investigadores (Schegloff, 1991; Sacks, 1992; Zimmerman, 1998; Antaki y Widdicombe, 1998; Stokoe y Smithson, 2001; Unamuno y Codó 2007), según los cuales la formación del sujeto no sería única y estática, es decir, que no serían sólo un conjunto de normas sociales las que establecerían la identidad de la persona, sino que la identidad se describe como una atribución negociable (durante el habla), variable y flexible.

Para analizar la complejidad de los procesos de construcción de la identidad durante el habla, Sacks (1992) desarrolló la idea de las categorías, dispositivos que llevan implícito el conocimiento de la sociedad y la cultura por parte de sus miembros. Estas categorías, aunque son consideradas como una unidad, pueden organizarse de forma duplicada (madre-hijo, experto-inexperto), ya que existen entre ellas relaciones de derechos y obligaciones. Por eso mismo, es importante analizar los procesos de categorización entre los hablantes, ya sean las auto-categorizaciones o las heterocategorizaciones (Unamuno y Codó, 2007), puesto que hay que ver las consecuencias que pueden tener unas sobre otras. Como dice Zimmerman (1998) las prácticas 
categorizadoras son algunos de los mecanismos mediante los cuales se ejerce el poder en la interacción y se efectúa en control y la inclusión o la exclusión social. Así, cuando se inicia una acción, una de las partes asume una identidad particular y proyecta una identidad recíproca para el o los co-participantes que, a su vez, pueden aceptar o no esa identidad.

\section{Language Brokering y conciencia metalingüística}

Son varios los investigadores que se han interesado en estudiar a estos chicos y chicas de primera o segunda generación de inmigrantes que adquieren una función primordial para la comunicación entre sus progenitores y otros miembros de la sociedad (Tse, 1996; Buriel, Perez, DeMent, Chavez, y Moran, 1998; Orellana, 2003; Love y Buriel, 2007; Umaña-Taylor 2003, en Morales y Hanson, 2005). Nombrados por Tse (1996) como language brokers $^{2}$ y por Orellana (2003) como parafraseadores, son definidos como hijos e hijas de familias de inmigrantes que traducen e interpretan para sus familias, miembros de su familia extensa, profesores, vecinos u otros adultos.

Aunque no existe un consenso entre los investigadores sobre todos los aspectos $\mathrm{y}$ efectos que tiene el language brokering en estos chicos y chicas, sí que podemos nombrar unas características comunes de los language brokers: (1) empiezan a serlo entre los 8 y 12 años; (2) normalmente, suele ser el hijo o hija mayor; y (3) mayoritariamente, son chicas (Morales y Hanson, 2005). Este último punto no se justifica directamente como una función que se da por el género, sino como causa de la naturaleza de las relaciones familiares (Orellana, 2003), ya que quien pasa más tiempo con la madre es quien más le traduce.

Tampoco no hay un acuerdo a la hora de determinar si el language brokering tiene consecuencias positivas o negativas en estos sujetos. Por un lado, en algunos estudios (Buriel et al., 1998), parece afectar a las dinámicas familiares creando un cambio en las relaciones de autoridad intergeneracional y tensiones entre madres e hijas, sobretodo a partir de la adolescencia. Además, a nivel intrapersonal, estas experiencias pueden crear en el language broker estrés y exceso de responsabilidad (Tse, 1996) y ello puede afectar en el desarrollo de su identidad por el hecho de tener que asumir el papel de adulto (Umaña-Taylor 2003, en Morales y Hanson, 2005). Por otro lado, estas interacciones con dos lenguas y dos culturas pueden tener como consecuencia una 
elevada confianza en las relaciones sociales y el desarrollo de recursos para resolver problemas (Buriel et al., 1998), así como también que sean personas más maduras e independientes (Tse, 1996). Según Orellana (2003), los language brokers, por el hecho de traducir, tienen capacidades bilingües y habilidades de lecto-escritura sofisticadas. Se habla pues de estos chicos y chicas como sujetos con habilidades cognitivas más altas y que les permiten ser más conscientes del funcionamiento de sus lenguas.

Varias investigaciones sobre bilingüismo y plurilingüismo van en la misma línea. Según algunos estudios (Bialystok, 2001, 2007; Bialystok, McBride-Chang y Luk, 2005), las personas bilingües tienen algunas habilidades más desarrollados que las personas monolingües (formación de conceptos, flexibilidad, control de la atención, entre otros) y una mejor conciencia metalingüística, además de desarrollar una orientación más analítica hacia el lenguaje (Vygotsky, 1962, en Cummins y Swain 1986; Cook, 2002). Todo esto es relevante en este estudio ya que las participantes son niñas y jóvenes plurilingües de primera o segunda generación inmigrante de edades comprendidas entre los doce y los dieciséis años que son susceptibles de ser language brokers.

\section{Participantes}

Como se ha comentado anteriormente, los datos para este estudio se recogieron en dos centros educativos de Barcelona, uno de educación primaria y uno de educación secundaria, ambos centros donde existe un porcentaje de alumnado de origen inmigrante muy elevado, en gran medida, procedente de Pakistán y de Marruecos. Las participantes del estudio son dieciséis, de las cuales cuatro son de educación primaria (once y doce años), diez son de educación secundaria (de catorce a dieciséis años) y dos son las madres de dos alumnas de secundaria. ${ }^{3}$ Antes del inicio de la investigación no existía ninguna relación entre la investigadora y las participantes. La información sobre las participantes se muestra en la siguiente tabla:

Tabla 1. Participantes

\begin{tabular}{c|c|c|c}
\hline & Participante & $\begin{array}{c}\text { País de origen } \\
\text { propio o familiar }\end{array}$ & $\begin{array}{c}\text { Tiempo de estancia en España } \\
y / o \text { Cataluña }\end{array}$ \\
\hline \multirow{2}{*}{$\begin{array}{c}\text { Educación } \\
\text { Primaria } \\
12 \text { años }\end{array}$} & Ikram & Marruecos & Nacida en Cataluña \\
\cline { 2 - 4 } & Hanna & Marruecos & Nacida en Cataluña \\
\cline { 2 - 4 } & Rashida & Marruecos & Nacida en Cataluña \\
\cline { 2 - 4 } & Mariam & Marruecos & Nacida en Cataluña \\
\hline
\end{tabular}




\begin{tabular}{l|c|c|c}
\hline Educación & Razia & Bangladesh & Nacida en Cataluña \\
\cline { 2 - 4 } Secundaria & Kamila & Pakistán & Nacida en Cataluña \\
\cline { 2 - 4 } 14-16 años & Farida & Marruecos & 3 años \\
\cline { 2 - 4 } & Maria & Pakistán & 2 años \\
\cline { 2 - 4 } & Nawal & Pakistán & 2 años \\
\cline { 2 - 4 } & Nida & Pakistán & 2 años \\
\cline { 2 - 4 } & Munira & Pakistán & 1 año y 10 meses \\
\cline { 2 - 4 } & Hajrah & Pakistán & 1 año y 6 meses \\
\cline { 2 - 4 } & Zareen & Pakistán & 1 año y 6 meses \\
\cline { 2 - 4 } & Nour & Marruecos & 10 meses \\
\hline \multirow{7}{*}{ Madres } & Madre & Marruecos & 3 años \\
& Farida & & \\
\cline { 2 - 4 } & Madre & Pakistán & 14 años \\
\hline & Kamila & & \\
\hline
\end{tabular}

\section{Datos}

Los datos analizados en este estudio proceden de dos fuentes:

1. Un conjunto de entrevistas (focus group) que se basan en un formato semiestructurado (Cots y Nussbaum, 2008), es decir, entrevistas de formato libre (entrevista-conversación), pero guiadas por una serie de preguntas (protocolo preparado con anterioridad) para indagar sobre datos biográficos, bagaje lingüístico, elección de lenguas en diferentes contextos, representación de la sociedad de recepción, ideas sobre el futuro, relevancia atribuida a las lenguas autóctonas (catalán y castellano) y sobre si las niñas y jóvenes son o no maestras de lengua de sus madres. Estas interacciones semiestructuradas se basan en la idea de la entrevista 'activa', aquella en la cual la entrevistadora también contribuye a la co-construcción del contenido $\mathrm{y}$, por consiguiente, su comportamiento tiene que formar parte del análisis (Mann, 2010, Unamuno y Nussbaum, 2005).

Dado que son muy comunes las interacciones plurilingües en el contexto de estudio, no se estableció una lengua para las entrevistas, es decir, no hubo una negociación explícita sobre qué lengua se iba a hablar. La investigadora hablaba, durante la mayor parte de la entrevista, en catalán o bien cambiaba al castellano y las participantes en castellano, en catalán y/o en la lengua que se hable en su entorno familiar. En este sentido, las entrevistas se desarrollan de forma plurilingüe $\mathrm{y}$ basadas en la intercomprensión.

2. Dos actividades que sirvieron como pretexto para la discusión sobre las categorizaciones y la SL: 
a. Mis lenguas: esta actividad, aplicada con el propósito de descubrir las biografías y las competencias lingüísticas que dicen tener las participantes e iniciar un diálogo sobre las lenguas, se realizó con las diez jóvenes participantes de secundaria. Se trataba de dibujar un cuerpo humano (de forma esquemática) y situar cada una de las lenguas que se conoce (de forma activa o pasiva) en una parte del cuerpo según qué significado tenga para uno o lo que se piense de esta lengua. Así, la lengua materna o la lengua que se siente más cercana se suele poner en el corazón o la que se cree más útil, en la mano, por ejemplo.

b. Preparación de entrevista: dos de las participantes de secundaria, Kamila y Farida, prepararon una entrevista para sus madres con la premisa de que tenía que servir para que la investigadora pudiera conocer a sus madres como hablantes de lenguas. Únicamente estas dos participantes prepararon esta entrevista y las aplicaron a sus madres: una fuera del entorno escolar (Farida) y la otra durante una entrevista con la investigadora, ella misma (Kamila) y su madre en el entorno escolar.

A partir de estas dos actividades, se desarrollaron conversaciones sobre las lenguas, en las cuales se pueden analizar las categorizaciones y la SL de las participantes.

\section{Metodología de análisis de los datos}

Los datos seleccionados, transcritos y tratados para el análisis que se realiza en este estudio son fragmentos de las conversaciones. El enfoque del análisis es de tipo cualitativo y basado en el análisis conversacional (Sacks, 1992). Según Schegloff (1991), éste sería el enfoque adecuado para estudiar las categorías, puesto que tienen que ser 'descubiertas' a través del estudio de la interacción social. Este enfoque nos permite estudiar las categorizaciones (auto-categorizaciones y hetero-categorizaciones) de las participantes en las interacciones. Como indican Schegloff (1991), Zimmerman (1998) y Antaki y Widdicombe (1998), el análisis conversacional se interesa por la perspectiva de los participantes sobre la organización social, para descubrir las categorías sociales y las identidades a través de la actuación (performance). 


\section{Resultados}

Competencias lingüisticas y conciencia metalingüistica

Los datos muestran la realidad multilingüe de las niñas y jóvenes participantes en el estudio. Tanto las alumnas de primaria como de secundaria aseguran tener en su repertorio lingüístico la lengua o lenguas familiares y las lenguas locales (castellano y catalán) y, en algún caso, otras lenguas que han aprendido en su país de origen o en la escuela. La competencia que tienen en lengua catalana y castellana depende del tiempo de escolarización en Cataluña, aunque los datos muestran que efectivamente se trata de sujetos plurilingües que pueden usar, como mínimo, tres lenguas a las cuales dan un valor y un uso diferente, como veremos a continuación. La lengua vehicular en casa con los progenitores es, habitualmente, una de las lenguas del país de origen (árabe, punyabí, urdú, hindi, bengalí) y, en algunas ocasiones, el castellano, pero niegan el uso del catalán. Según los datos, el uso de la lengua de origen en casa está relacionado con la voluntad de continuidad de la transmisión de las lenguas por parte de los progenitores. Asimismo, es la lengua castellana la que usan las jóvenes que han nacido o que hace más tiempo que viven en Cataluña para comunicarse con sus hermanos o compañeros de clase. Lo mismo ocurre en el barrio, es decir, que la lengua franca local es el castellano, como ya han demostrado numerosos estudios realizados en Cataluña en contextos similares (Unamuno y Nussbaum, 2006, Unamuno y Codó, 2007, Corona, Nussbaum y Unamuno 2012).

En lo relativo a la escuela, tanto las participantes de primaria como las de secundaria asocian el catalán a este contexto y a una necesidad para la educación, el progreso y el éxito laboral. De todos modos, aunque este es un hecho expresado por las participantes de forma generalizada, hay diferencias en el uso de la lengua según la edad durante las entrevistas. El resultado del análisis de los turnos de los dos grupos de participantes (de primaria y de secundaria) muestra que se da un mayor uso del catalán en el grupo de alumnas de primaria (como señalan también otros estudios como el de Nussbaum y Unamuno, 2006), un $73 \%$ de turnos en catalán y $27 \%$ en castellano en los datos de primaria, frente a un $24 \%$ de turnos en catalán y $76 \%$ en castellano en secundaria. La presencia del catalán en la escuela secundaria entre el alumnado inmigrante o de segunda generación parece que quedaría relegada a la comunicación con la comunidad educativa y, específicamente, a la clase de lengua catalana. 


\section{FRAGMENTO 1}

Participantes: Investigadora (INV), Farida (adolescente de origen marroquí, FAR), Kamila (adolescente de origen pakistaní, nacida en Cataluña, KAM)

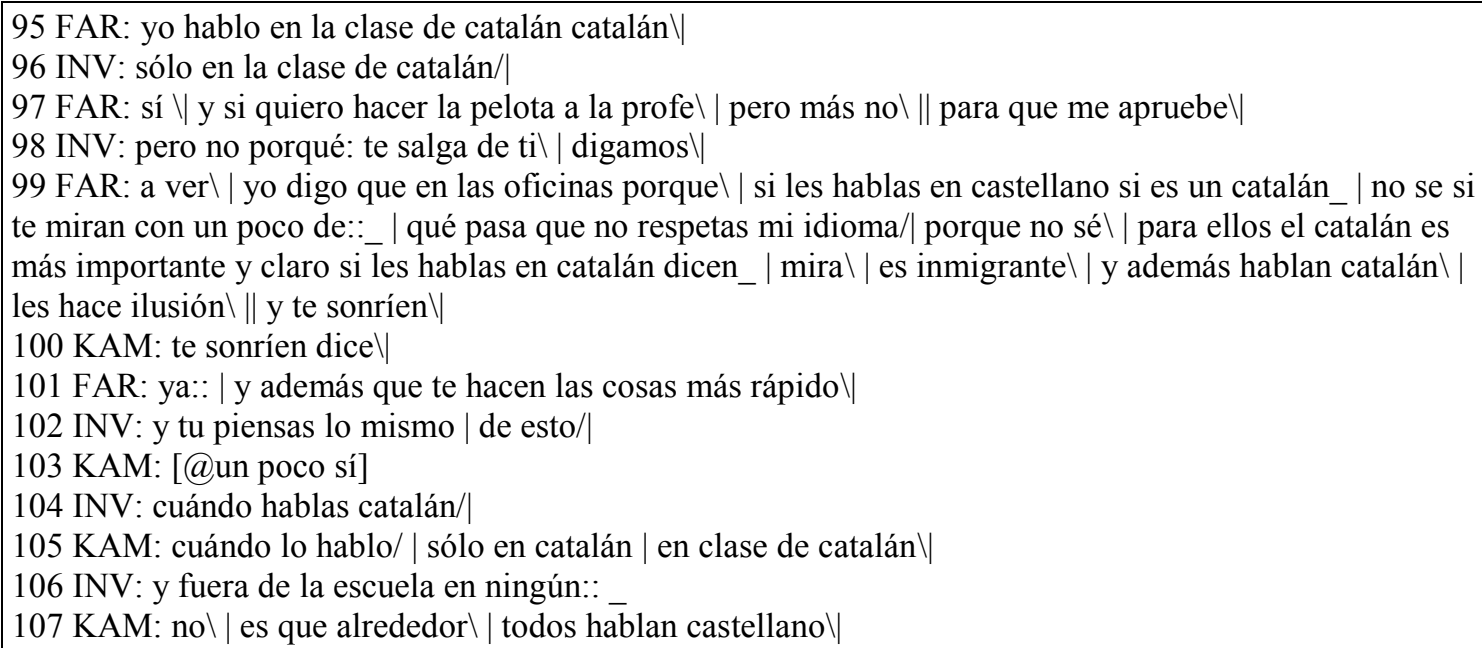

Como vemos en este fragmento, tanto KAM como FAR afirman hablar la lengua catalana sólo en la clase de catalán. De todos modos, el uso que hacen de esta lengua es más extenso, ya que, ciertamente, la mayoría de actividades de lectoescritura se desarrollan en catalán en el centro. En la actividad de preparación de la entrevista para sus madres que realizaron dos de las participantes (Kamila y Farida), la investigadora empezó hablando catalán, pero las participantes usaban, casi siempre, el castellano. Sin embargo, aunque evitaran el uso oral del catalán, en el momento de formular las preguntas y escribirlas, y sin que se les hubiera pedido de forma explícita, utilizan la lengua catalana. Parece pues que esta es la lengua que consideran propia de las actividades de lectoescritura de la escuela, como aparece también en estudios anteriores (Nussbaum y Cots, 2011).

\section{Las competencias lingüísticas de las madres}

Los datos parecen mostrar que la competencia lingüística en castellano, tanto de la madre de Kamila como de la madre de Farida, es muy baja. Aunque la madre de KAM lleva catorce años en Cataluña no tiene mejor competencia en lengua castellana que la madre de FAR que lleva tres años, sino lo contrario. Por lo tanto, el factor tiempo de estancia en el lugar no conlleva directamente mayor competencia lingüística de la/s lengua/s local/es, dado que hay otros factores que parecen influir. 
Por un lado, el factor trabajo se hace relevante a la hora de hablar del aprendizaje de la lengua de las mujeres inmigrantes. Esto podemos verlo en el caso de la madre de FAR que trabaja fuera de casa desde que llegó y tuvo que aprender un poco de castellano. En cambio, la madre de KAM nunca ha trabajado fuera de casa y su SL se ha visto más limitada. Por otro lado, no es el trabajo el factor determinante para hablar de aprendizaje de lengua/s por parte de las mujeres inmigrantes sino que tiene un peso más importante el entorno en el que viven.

FRAGMENTO 2

Participantes: Farida (adolescente de origen marroquí, FAR), madre de Farida (mujer de origen marroquí, MA_F)

1 FAR: por qué no hablas castellano/|

2 MA_F: yo cuando vine aquí no tengo::: || amigos castellano $\mid$ no tengo:: nada | vine con familia árabe_ para la calle habla árabe $\backslash$ | la televisión árabe $\backslash$ todo árabe $\backslash \mid$ para esto no yo no sabe hablar castellano $\backslash$ 3 FAR: por qué te cuesta hablar:: español/|

4 MA_F: porque yo no sabe hablar $\backslash \|$ no sabe leer $\backslash$ no sabe escribir $\backslash \|$ para eso yo no sabe hablar bien $\backslash$

Según dice la madre de Farida, no le hizo falta aprender la lengua para poder vivir en el entorno al cual llegó: en la calle podía comunicarse en árabe, la televisión la veía en árabe y, por lo tanto, parece que sus relaciones y actividades sociales no incluían la lengua castellana ni la catalana. Lo mismo le sucede a la madre de Kamila, ya que una de las lenguas de su barrio (Raval) es el urdú. Su SL en lengua castellana (no catalana) queda reducida a situaciones muy limitadas y concretas, como, por ejemplo, ir a las tiendas y preguntar precios. Además, en estas situaciones ambas han tenido y tienen a sus hijas para hacerles de mediadoras lingüísticas (language brokers) si lo necesitan.

\section{La conciencia metalingüistica y las nuevas competencias}

En el análisis de los datos, se detectan algunos aspectos relacionados con la conciencia metalingüística y la capacidad de análisis de la lengua que las alumnas han desarrollado. Por un lado, se sugiere un cierta conciencia fonológica de las lenguas que conocen. Durante la entrevista con las participantes de primaria, éstas explican que cantan una canción, originariamente en lengua castellana, para imitar la lengua que hablan sus madres, a la cual denominan hispamarroc. Podemos ver este fenómeno de estilización de la lengua en el siguiente fragmento. 


\section{FRAGMENTO 3}

Participantes: Ikram (niña de origen marroquí, nacida en Cataluña, IKR), Hannah (niña de origen marroquí, nacida en Cataluña, HAN), Rashida (niña de origen marroquí, nacida en Cataluña, RAS)

IKR, HAN, RAS: +a tu ladu mi sintu siguru $\backslash$ | a tu ladu no dudu $\backslash \mid$ a tu ladu yo puidu $\backslash$ vular $\backslash \mid$ a tu ladu mi sintu [@siguru] $\backslash \mid$ a tu ladu mi suinio $\backslash \mid$ se hará pur fin rialidad $\backslash$ |+ [fragmento cantado con acento árabe]

Ikram, una de las participantes, explica cómo habrían creado la canción: cambiando algunas palabras e imitando cómo hablan sus madres. Pero, esta actividad parece ser un ejercicio de conciencia metalingüística, y sobretodo fonológica, ya que las niñas aplican a la lengua castellana la fonética de la lengua árabe. Esta lengua, fonéticamente, sólo tiene tres fonemas vocálicos $(\mathrm{a}, \mathrm{i}, \mathrm{u})$ que pueden ser largos o cortos y, según en qué posición se encuentren, se convierten en semivocales. Si nos referimos al sistema consonántico, no existen fonemas consonánticos oclusivos [p], [g], (Sánchez, 2003). Así, las participantes, cuando cantan la canción, cambian los sonidos vocálicos que no forman parte de la fonética árabe por [i] o por [u]. En las consonantes, se producen modificaciones en la $[\mathrm{g}]$, que se transforma en una nueva consonante que es una mezcla entre $[\mathrm{g}]$ y $[\mathrm{k}]$ (por ejemplo, en la palabra siguro), y en la [n], que se cambia por [n], y también en las semi-consonantes (suinio, en lugar de sueño).

La realidad sociolingüística del contexto de estudio provoca que estas jóvenes tengan que llevar a cabo diversas actividades que podrían potenciar una mejora de su competencia metalingüística y hacer que desarrollen una competencia traductora y una competencia docente. Como indican los datos, las chicas que tienen un mayor dominio de las lenguas autóctonas afirman hacer de language brokers de sus madres en los ámbitos sanitario, escolar, administrativo y laboral. Esta labor de acompañante y traductora de la madre pertenece, según los datos, a la hija mayor y no a las menores. Aún así, en la entrevista con KAM, su madre y la investigadora, era KAM (la hija menor) quien le traducía las partes del discurso que no entendía. Haría falta un estudio más a fondo para determinar cómo se reparten estas funciones en las familias de origen inmigrante. En cambio, la tarea de docente de lengua parece que es generalizada tanto en primaria como en secundaria e independiente de la edad o la posición en la familia. Esta actividad docente comprende: la corrección del discurso, la ayuda con los deberes, 
la conversación con las madres, la co-construcción del discurso y la traducción. La función que realizan las hijas es, por tanto, de agentes transmisoras de lengua.

\section{Procesos de categorización e identidades en flujo}

En el caso de las alumnas inmigrantes o de segunda generación, parece tener gran importancia la adscripción a una o a otra lengua para definir su identidad. Tanto FAR como KAM se presentan como hablantes plurilingües y con un conocimiento sociolingüístico elevado del lugar donde viven: el catalán es la lengua de la escuela, de las actividades de lectoescritura y la lengua "de los catalanes", el castellano es la lengua franca del barrio donde viven y la lengua de comunicación con amigos y hermanos y la lengua o lenguas de los progenitores es la lengua de origen que no hay que perder. No se trata sólo de la lengua que se hable sino que lo relevante parece ser el hecho de hablarla bien o no y las consecuencias que eso pueda tener.

FRAGMENTO 4

Participantes: Farida (adolescente de origen marroquí, FAR), Kamila (adolescente de origen pakistaní, nacida en Cataluña, KAM), Investigadora (INV)

147 FAR: a ver es que lo habla raro $\backslash$ | porque no sabe hablar $\backslash$ | entonces en lugar de decir _ yo sé | dice yo saber》

148 todas: [risa]

149 FAR: entonces es divertido $\backslash$

150 KAM: para mi no es divertido_| yo le:: yo le regaño $\mid$

151 FAR: ah para mi es divertido | no sé $\mid$

152 INV: le regañas/|

153 FAR: a ver tampoco o sea_|

154 KAM: pero luego la gente va a imitarte y luego se burlan $\backslash$

155 FAR: y qué/ | a mi me da igual que me llamen inmigrante $\backslash$ si al final y al cabo yo soy inmigrante $\mid$

Las actitudes hacia la categorización de cómo hablan la lengua local las madres de las jóvenes es muy diferente en el caso de FAR y de KAM. FAR categoriza a su madre como a una persona que habla mal la lengua local y se construye el discurso junto con las otras participantes, que confirman esta categorización a través de la risa. Además, FAR relaciona el hecho de hablar la lengua local mal y las burlas ajenas con la categoría "inmigrante", categoría que aparece por primera vez en el discurso. Esta autocategorización de FAR como inmigrante no es negada por las participantes, lo cual parece mostrar que este grupo de jóvenes asume la identidad de "inmigrante".

Pero, KAM rechaza el hecho de que pueda ser divertido ser incluida dentro de este grupo social y lucha contra ello. Esta diferencia podría darse por el hecho de que 
KAM es inmigrante de segunda generación. La identidad, según esta participante, está totalmente unida a la lengua que hable la persona y cómo la hable $\mathrm{y}$, por lo tanto, es importante aprender la lengua franca para funcionar en la sociedad, para socializarse lingüísticamente. La lengua adquiere un valor identificatorio efectivo, puesto que aparece como una herramienta para calificar a la persona a través del discurso y en la interacción con los interlocutores. El cambio de lengua tiene también, en este sentido, una función identitaria, aunque la identidad se presenta en muchos casos como fragmentada, es decir, construida en cada situación social.

El hecho de que las jóvenes sean las responsables, en la mayoría de los casos, de hacer de traductoras (language brokers) y maestras de sus madres parece tener consecuencias: (1) en el lugar en el que se sitúan ellas mismas y su propia imagen y (2) en la imagen que tienen de sus madres. Las auto-categorizaciones de las hijas se refieren a las categorías de experta-inexperta de lengua $\mathrm{y}$, de esta manera, se sitúan en una posición de responsables de sus madres y de la transmisión de la lengua local.

\section{FRAGMENTO 5}

Participantes: Investigadora (INV), Farida (adolescente de origen marroquí, FAR)

75 INV: i tu | com et sents | quan li tradueixes tot/

76 FAR: sóc gran

$77 \mathrm{INV}$ : què vols dir que ets gran/|

78 FAR: Que:: m:::: || responsable $\backslash$ perquè: $\backslash$ si no le traduzco no entiende claro || hay que traducirle $\mid$

79 INV: però t'agrada fer això de | d'haver-la d'ajudar:: i::

80 FAR: hombre $\backslash$ | a una madre siempre::_| no se::_| es tu madre $\backslash||$ si fuera otra persona es otro tema $\backslash$ pero como que es mi madre_| pues_l

81 INV: i::_ || això ho fas des del principi que vas venir aquí \ no//

82 FAR: sí::!|

83 INV: d'ajudar-la:: | de vull dir_| d'acompanyar-la si ha d'anar al metge fer la traducció i aixíl|

84 FAR: sí $\backslash$ y de hecho por por eso intentaba aprender el castellano antes $\backslash$

Traducción

75 INV: y tu | cómo te sientes | cuando le traduces todo/

76 FAR: soy mayor

77 INV: qué quieres decir con soy mayor/|

78 FAR: Que:: m:::: || responsable $\backslash$ porque: $\vee$ si no le traduzco no entiende claro $\|$ hay que traducirle $\backslash$

79 INV: pero te gusta hacer esto de | tener que ayudarla:: y::

80 FAR: hombre $\backslash$ | a una madre siempre::_| no se::_| es tu madre $\backslash||$ si fuera otra persona es otro tema $\backslash$ pero como que es mi madre_| pues_l

81 INV: y::_ || esto lo haces desde el principio que viniste aquí $\backslash$ no/|

82 FAR: sí::||

83 INV: de ayudarla:: $\backslash$ | de quiero decir_| de acompañarla si tiene que ir al médico hacer la traducción y así $\mid$

84 FAR: sí $\backslash$ y de hecho por por eso intentaba aprender el castellano antes $\backslash$ 
Vemos como FAR se sitúa en una posición de experta de lengua y a su madre como dependiente de su ayuda. Según comenta, aprendió la lengua castellana antes por el hecho de que su madre necesitaba que la acompañara y fuera su traductora/mediadora y se sentía responsable de la socialización de su madre. Ciertamente, se produce un cambio de rol familiar y de la dirección de la transmisión de lengua en estas jóvenes de origen inmigrante. Esta auto-categorización de expertas tiene como consecuencia la hetero-categorización de las madres como hablantes inexpertas de la lengua local. En general, esta hetero-categorización aparece con risa, no solamente por parte de las jóvenes, sino que, según dicen, es todo el núcleo familiar que se ríe de cómo hablan las madres. Éstas son presentadas como sujetos no integrados lingüísticamente y las sitúan en un lugar discriminado y subordinado de la sociedad y, en ciertos aspectos, también de la familia. La lengua que hablan se describe como "novato, saben decir lo básico y no es como tener una conversación de esas”. Esta hetero-categorización de inexpertas se impone a las madres por parte del núcleo familiar y, según dicen, por la sociedad de acogida; esto tiene como consecuencia la auto-categorización de las madres como inexpertas de lenguas, como dependientes en lo que se refiere al uso de la lengua local y la exclusión lingüística y de comunicación en ciertos momentos y ámbitos.

Según los datos, parecería que la SL de las hijas podría influir en la forma cómo se da la SL de sus madres; es decir, si la hija identifica el contexto barrio con la lengua castellana o la escuela con la lengua catalana y con las prácticas lecto-escritoras, la madre podría aprenderlo de la misma manera.

\section{FRAGMENTO 6}

Participantes: Investigadora (INV), madre de Kamila (mujer de origen pakistaní, MARE), Kamila (adolescente de origen pakistaní, nacida en Cataluña, KAM)

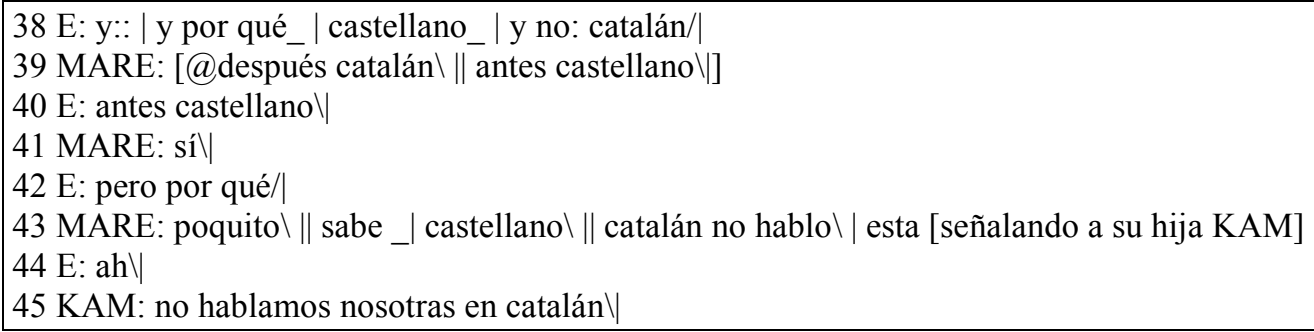


La madre de KAM confirma este hecho: aprende castellano y no catalán porque sus hijas hablan entre ellas castellano. Seguramente, esto está relacionado con el hecho de que la lengua franca del barrio es el castellano y, por lo tanto, a parte del valor ideológico que las hijas den a las lenguas, también influye en su SL una cuestión práctica. Por otro lado, las hijas se presentan en todo momento como co-constructoras del conocimiento de sus madres: (1) son sus traductoras y, por lo tanto, las que las acompañan en su socialización en los diferentes contextos; y (2) son sus maestras de lengua y las que co-construyen el discurso con ellas.

\section{Discusión}

El análisis de los datos del estudio muestra diferentes aspectos interesantes en lo que se refiere al aprendizaje de lenguas por parte del colectivo inmigrante en Cataluña y su situación en la sociedad, aspectos que deberían considerarse en el campo de la enseñanza. La categorización negativa de las madres parece que tiene dos consecuencias: (1) para las hijas, ya que el hecho de auto-categorizarse como expertas de lengua y la función que adquieren de traductoras y maestras de lengua de sus madres provoca en ellas un sentimiento de responsabilidad hacia sus madres. En algún caso, parece que esta responsabilidad hace que se de más rápidamente el aprendizaje de las lenguas locales. Son, por lo tanto, las hijas quienes tienen la posibilidad de aprender la lengua, de socializarse lingüísticamente y de asumir el papel de transmisoras de lengua. (2) Para las madres, puesto que el hecho de auto-categorizarse como inexpertas de lengua muestra que creen muy poco en sus posibilidades como hablantes competentes. El análisis parece mostrar que se da un cambio en la función de los miembros de las familias y de la transmisión de lengua y aparecen los roles de "experto-inexperto" como variables en la interacción y en las circunstancias. Así, las hijas son las expertas de lengua en lo que se refiere a las lenguas locales pero las madres lo son en la lengua o lenguas de origen. Además el papel de transmisoras de lengua de origen de las madres es fundamental para la mantención de la lengua de origen y, por lo tanto, para la existencia del entorno plurilingüe.

Los resultados sobre la influencia en la SL de las madres por parte de las hijas y, por lo tanto, el cambio de dirección en la transmisión intergeneracional de lenguas muestran que se trata de una nueva situación social en la cual, a causa del hecho 
migratorio, estos grupos sociales tienen que socializarse lingüísticamente de nuevo, cosa que conlleva nuevos fenómenos lingüísticos, sociales y familiares.

Este estudio pretende ser el inicio de un trabajo más amplio de exploración de las especificidades de la dirección de la SL de la población de origen inmigrante y es necesaria más investigación sobre estos aspectos para confirmar los resultados expuestos en este texto.

\section{Referencias bibliográficas}

Antaki, C., y Widdicombe, S. (Coords.) (1998). Identities in talk. London, Thousand Oaks, New Delhi: SAGE Publications.

Bayley, R., y Schecter, S. (2003). Language and socialization in bilingual and multilingual societies. Clevendon, Buffalo, Toronto, Sydney: Multilingual Matters LTD.

Bialystok, E. (2001). Bilingualism in development. Language, literacy and cognition. USA: Cambridge University Press.

Bialystok, E. (2007). Acquisition of literacy in bilingual children: A framework for research. Learning language (57, Suppl. 1, june 2007): 45-77.

Bialystok, McBride y Luk. (2005). Bilingualism, language proficiency, and learning to read in two writing systems. Journal of educational psychology 97, 580-590.

Buriel, R., Perez, W., DeMent, T.L., Chavez, D.V., y Moran, V.R. (1998). The relationship of language brokering to academic performance, biculturalism, and self efficacy among Latino adolescents. Hispanic journal of behavioral sciences, 20(3), 283-297.

Butler, J. (1990). Gender trouble. Feminism and the subversion of identity. NY y London: Routledge.

Cook, V.J. (2002). Background to the L2 user. In V.J. Cook (Ed.) Portraits of the L2 user (pp. 1-28). Clevedon: Multilingual Matters.

Corona, V., Nussbaum, L. y Unamuno, V. (2012). The emergence of new linguistic repertoires among Barcelona's youth of Latin American origin. International journal of bilingual education and bilingualism, 16(2), 182-194. Special Issue: Catalan in the 21st century.

Cots, J.M., y Nussbaum, L. (2008). Communicative competence and institutional affiliation: Interactional processes of identity construction by immigrant students in Catalonia. International journal of multilingualism, vol. 5-1 pp. 17-40.

Cummins, J., y Swain, M. (1986). Bilingualism in education: Aspects of theory, research and practice. London y New York: Longman.

Fisher, J.L. (1970). Linguistic socialization: Japan and the United States. In Hill, R. y König, R. (eds) (1970). Families in the East and West. Socialization process and kinship ties (pp. 107-108). Paris y The Hague: Mouton y Co.

Kulick, D., y Schieffelin, B. (2004). Language socialisation. In A. Duranti (Ed) A Companion to linguistic anthropology (pp. 88-119). USA, UK y Australia: Blackwell Publishing.

Lakoff, R. (1975). Language and woman's place. New York y London: Harper y Row Publishers.

Love, J. y Buriel, R. (2007). Language brokering, autonomy, parent-child bonding, 
biculturalism, and depression: A study of Mexican American adolescents from immigrant families. Hispanic journal of behavioral sciences, 29:472.

Mann, S. (2010). A critical review of qualitative interviews in applied linguistics. Applied linguistics 2011 (32/1), pp. 6-24. Oxford University Press.

Miller, E. (2012). Performativity theory and language learning: Sedimentating, appropriating and constituting language and subjectivity. Linguistics and education (23): 88-99.

Morales, A., y Hanson, W. (2005). Language brokering: An integrative review of the literature. Hispanic journal of behavioral sciences, 27(4), 471-503.

Nussbaum, L., y Cots, J-M. (2011). Doing learning languages in a multilingual context: Pragmatic aspects of classroom discourse in Catalonia. En L. Payrató y J.M. Cots (Coords.) The pragmatics of catalan (pp. 331-359). Londres: De Gruyter.

Nussbaum, L., y Unamuno, V. (Coords.) (2006). Usos i competències multilingües entre escolars d'origen immigrant. Bellaterra: Servei de Publicaciones de la Universitat Autònoma de Barcelona.

Ochs, E., y Schieffelin, B. (1982). Language Acquisition and socialization: Three developmental stories and their implications. Working papers in sociolinguistics (105). Austin, Texas : Southwest Educational Development Laboratory.

Orellana, M.F. (2003). Responsibilities of children in Latino immigrant homes. New directions for youth development, 100, 25-39.

Sacks, H. (1992). The baby cried, the mommy picked it up. En: Jefferson (Ed.) (1992) Lectures on conversation. Blackwell Publishing.

Schegloff, E. (1991). Talk and the Social Structure. En: Boden, D. y Zimmerman, D. (Coords.) Talk and social structure: Studies in ethnomethodology and conversation analysis, Cambridge: Polity Press in association with Blackwell, 44-70. University of California Press.

Schieffelin, B., y Ochs, E. (1986). Language socialization. Annual review of anthropology (15):163-191.

Schieffelin, B., y Ochs, E. (1995). Language socialization across cultures. Studies in the social and cultural foundations of language (3). Cambridge: Cambridge University Press.

Stokoe, E.H., y Smithson, J. (2001). Making gender relevant: Conversation analysis and gender categories in interaction. Discourse and society 12(2): 243-269.

Tse, L. (1996). Language brokering in linguistic minority communities: The case of Chinese- and Vietnamese-American students. Bilingual research journal, 20(34). 485-198.

Unamuno, V., y Nussbaum, L. (2005). L'entrevista com a pràctica social i com a espai de construcció d'identitats. A M. Labarta (ed.) Approaches to critical discourse Analysis. València: Universitat de València.

Unamuno, V., y Nussbaum, L. (2006). De la casa al aula: ámbitos y prácticas de transmisión y aprendizaje de lenguas. Textos de didáctica de la lengua y de la literatura (42): 43-51.

Unamuno, V., y E. Codó (2007). Categorizar a través del habla: la construcción interactiva de la extranjeridad. Discurso y sociedad, 1 (1): 117-143.

Zimmerman, D. (1998). Identity, context and interaction. A: Antaki, C. y Widdicombe, S. (Ed.) Identities in talk. London, Thousand Oaks, New Delhi: SAGE Publications. pp. 87-106. 


\footnotetext{
${ }^{1}$ Los datos de la escuela de primaria fueron recogidos y cedidos para este estudio por Dra. Dolors Masats de la Universitat Autònoma de Barcelona.

${ }^{2}$ El término language broker lo traducimos como intermediario/a lingüístico/a.

${ }^{3}$ Teniendo en cuenta los resultados de los estudios previos sobre Language Brokering, los cuales apuntan al inicio de esta actividad entre los 8 y los 12 años y al hecho de que, mayoritariamente, son las chicas quienes tienen esta función, se eligieron estos centros para el estudio. Así, se necesitaban alumnas desde los 8 o 9 años hasta la educación secundaria, de origen inmigrante de primera o de segunda generación. El hecho que fueran, casi todas, de Marruecos o de Pakistan fue por el hecho de que son estas dos las nacionalidades mayoritarias de las familias inmigrantes del entorno y, por lo tanto, de estos centros.
}

\section{Referencia de la autora}

Júlia Llompart Esbert es investigadora predoctoral del grupo GREIP en la Universidad Autónoma de Barcelona. Fue profesora de lengua y cultura en la Universidad de Massachusetts (2009-2012) y, en septiembre del 2012, terminó el máster en Didáctica de la Lengua y la Literatura con la presentación de la tesina El cambio en la transmisión intergeneracional de lenguas: las nuevas maestras de lengua. Le interesan los nuevos procesos de transmisión de lenguas, la educación bilingüe y los procesos de construcción de la identidad.

Email: julia.llompart@gmail.com

\section{Anexo}

\section{Simbología de transcripción de datos}

- Pseudónimo de los hablantes

- Entonación ascendente

- Entonación descendente

- Entonación mantenida

- Pausa corta

- Pausa media

- Pausa larga

- Alargamiento silábico

- Encabalgamiento

- Enunciados pronunciados riendo

- Comentarios de quien transcribe

- Transcripción fonética aproximada

- Fragmentos incomprensibles

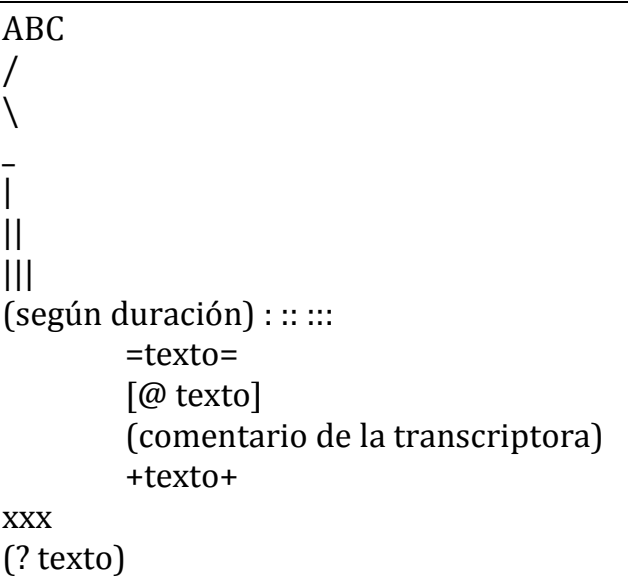

- Fragmentos dudosos

(? texto)

Para citar este artículo:

Llompart Esbert, J. (2013). De madres a hijas, de hijas a madres: El cambio en la transmisión intergeneracional de lenguas. Bellaterra Journal of Teaching \& Learning Language \& Literature, 6(3), 47-65. 